\title{
SEMI-AUTOMATIC GENERATION OF A CORPUS OF WIKIPEDIA ARTICLES ON SCIENCE AND TECHNOLOGY
}

\author{
Generación semi-automática de un corpus de \\ artículos de Wikipedia sobre ciencia y tecnología
}

Julià Minguillón, Maura Lerga, Eduard Aibar, Josep Lladós-Masllorens and Antoni Meseguer-Artola

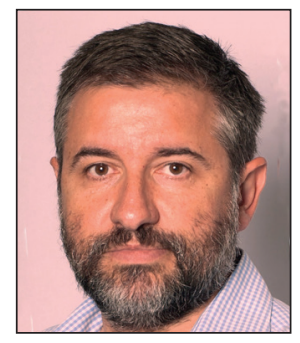

Julià Minguillón received his PhD degree from the Universitat Autònoma de Barcelona (UAB) in 2002. In January 2001 he joined the Universitat Oberta de Catalunya (UOC) faculty in the Computer Science, Multimedia and Telecommunication Studies Department. He is member of the UOC's Laika research group. His research interests include the uses of open educational resources, digital repositories, and social tools for teaching and learning in virtual learning environments, educational data mining, learning analytics, and data visualization.

http://orcid.org/0000-0002-0080-846X

Universitat Oberta de Catalunya Rambla Poblenou, 156. 08018 Barcelona, Spain jminguillona@uoc.edu

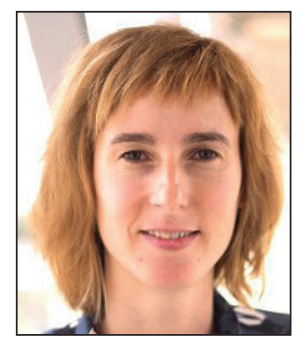

Maura Lerga, sociologist, holds a master's on Urban Anthropology. She is an experienced research assistant with a demonstrated history of working in the higher education field. She is skilled in sociology, data analysis, academic writing, strategic planning, and project management with a strong background as a social consultant. She is currently working at the International Center of the Universitat Rovira i Virgili (Spain), where she is in charge of four Erasmus+ projects, as project manager. She is a former member of the Open Science and Innovation Research Group (Universitat Oberta de Catalunya), where she was studying the scientific controversies in Wikipedia.

http://orcid.org/0000-0002-3516-0931

Universitat Oberta de Catalunya Avda. Tibidabo, 39-43. 08035 Barcelona, Spain mlergaf@uoc.edu

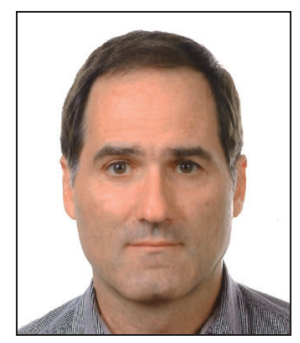

Eduard Aibar is an associate professor of Science and Technology Studies at the Arts \& Humanities Department, Universitat Oberta de Catalunya (UOC). He has been the director of the Internet Interdisciplinary Institute and vice-president for research at UOC. He leads a research group on open science and innovation. His research has focused on the interaction between scientific and technological development and organizational and social change in areas such as eGovernment, town planning, and the Internet. He is currently leading a research project on the relationship between science and peer production.

http://orcid.org/0000-0002-1727-1523

Universitat Oberta de Catalunya Avda. Tibidabo, 39-43. 08035 Barcelona, Spain eaibar@uoc.edu

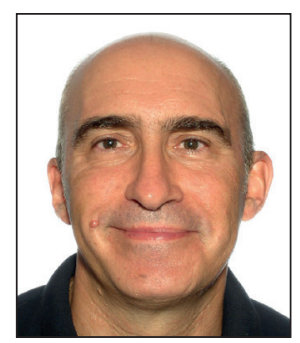

Josep Lladós-Masllorens is an associate professor of the Business and Economics Department at the Universitat Oberta de Catalunya (UOC), Barcelona (Spain). In 1998, he received a PhD in Business and Economics Science from the Universitat de Barcelona. He is a member of the Digital Business Research Group (DigiBiz), a consolidated research team recognized by the Generalitat of Catalonia. His research interests include economic geography, innovation systems, global value chains, and sharing economy; he is the author of several scientific papers, reports, and book chapters on these topics.

http://orcid.org/0000-0002-6236-5166

Universitat Oberta de Catalunya Avda. Tibidabo, 39-43. 08035 Barcelona, Spain jlladosm@uoc.edu 


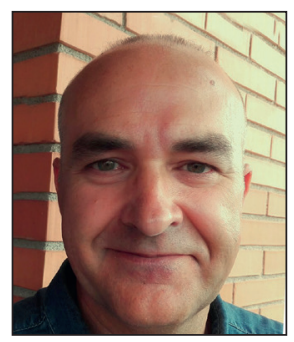

Antoni Meseguer-Artola has a doctoral degree in Economics and Business Sciences, a master's degree in Economic Analysis from the UAB, and a bachelor's degree in Mathematics from the UB. $\mathrm{He}$ is an associate professor of Quantitative Methods for Economics and Business at the UOC and a member of the DigiBiz research group. He is author of several articles and conferences on digital marketing, consumer behavior, game theory, and e-learning. He has a merit in research. He has also authored several teaching books in the field of mathematics and statistics. At the UOC, he has been director of the Bachelor's Degree Program in Work Sciences (2001-2006) and dean of the Faculty of Economics and Business (2006-2010).

http://orcid.org/0000-0002-7817-3695

Universitat Oberta de Catalunya Avda. Tibidabo, 39-43. 08035 Barcelona, Spain ameseguer@uoc.edu

\begin{abstract}
Despite the huge amount of scientific and technological content available on the World Wide Web, most of it is closed behind paywalls, as with academic journals, or almost invisible, as with institutional repositories. Wikipedia can act as a chain-transfer agent, providing people with an accessible, organized structure containing both understandable content and links to original sources. In Wikipedia, categories are collaboratively created and thus become a folksonomy rather than a true taxonomy. Consequently, categories are not a reliable tool to identify topics' organization. In this paper we describe a semi-automatic method, based on random walks, for determining a subset of pages containing scientific and technological content in the Spanish Wikipedia. Using the Unesco taxonomy, we determined the underlying graph structure of our corpus and detected clusters of pages strongly linked, establishing relationships between knowledge domains. Finally, we present the distribution of Wikipedia articles according to the Unesco taxonomy and the resulting map of scientific and technological content.
\end{abstract}

\title{
Keywords
}

Wikipedia; Science and technology; Corpus; Infomap; Community detection; Unesco taxonomy.

\section{Resumen}

A pesar de la gran cantidad de contenido científico y tecnológico disponible en la World Wide Web, su mayoría se encuentra encerrado tras sistemas de pago, como las revistas académicas, o es casi invisible, como los repositorios institucionales. Wikipedia puede actuar como un agente de transferencia, proporcionando una estructura organizada y accesible conteniendo tanto contenidos como enlaces a las fuentes originales. En Wikipedia las categorías se han cread colaborativamente y por lo tanto son más una folksonomía que una verdadera taxonomía. Consecuentemente, las categorías no son una herramienta válida para identificar la organización de los contenidos. En este artículo se describe un método semi-automático, basado en paseos aleatorios, para determinar un subconjunto de páginas con contenido científico y tecnológico de la Wikipedia española. Usando la taxonomía Unesco, se determina la estructura subyacente del grafo del corpus y se detectan grupos de páginas fuertemente enlazadas, estableciendo las relaciones entre las áreas de conocimiento. Finalmente, se presenta la distribución de artículos de Wikipedia de acuerdo con la taxonomía Unesco y el mapa resultante de contenido científico y tecnológico.

\section{Palabras clave}

Wikipedia; Ciencia y tecnología; Corpus; Infomap; Detección de comunidades; Taxonomía Unesco.

Minguillón, Julià; Lerga, Maura; Aibar, Eduard; Lladós-Masllorens, Josep; Meseguer-Artola, Antoni (2017). "Semi-automatic generation of a corpus of Wikipedia articles on science and technology". El profesional de la información, v. 26, n. 5, pp. 995-1004.

\section{Introduction}

Wikipedia has become one of the most popular websites in the World Wide Web. Due to its highly linked hypertext structure, it is a resource that is accessible through search engines, including Google (Ermann; Frahm; Shepelyansky, 2015). It is ranked in seventh position in the Internet rankings of Alexa, for instance. In fact, almost any search in Google returns one or more Wikipedia pages on the first results page, and often in first position, thus promoting its usage. Therefore, Wikipedia has rapidly become the most used reference source for any user searching for information about any topic (Ponzetto; Strube, 2007). On the other hand, Wikipedia has also become an excellent resource for research purposes (Medelyan et al., 2009), including both content and semantic analysis (Ryu; Jang; Kim, 2014), as it provides a huge source of organized human knowledge.

\section{Wikipedia has become the main plat-} form for the public communication of science 
A less well-known fact about Wikipedia is that it has also become the main platform for the public communication of science. Recent studies on communication and public perception of science prove that the number of people who rely on Wikipedia as a source of information about science and technology has rapidly increased in recent years and has surpassed all other media (Brossard; Scheufele, 2013; Snyder, 2013). Among students, Wikipedia is the main information source (Kim; Sin; Yoo-Lee, 2014). Even faculty and scientists themselves have become frequent users of Wikipedia in searching for scientific and academic issues (Aibar et al., 2015; Snyder, 2013).

Considering its increasing influence and use, the analysis of the scientific and technological content of Wikipedia in terms of quality, completeness, reliability and bias, to mention just a few aspects, is of great interest for many fields including science studies, public understanding of science, information management, etc. But for that analysis a previous step is needed: the identification of the corpus of Wikipedia articles dealing with science and technology issues. This is indeed the problem we have addressed in this paper and, for the reasons we will now show, it proves to be a non-trivial and hard task.

\section{The Spanish language edition of} Wikipedia has more than 1.2 million articles and is the $9^{\text {th }}$ largest, with more than 15,000 active users

Regarding its content, Wikipedia includes more than five miIlion articles in its English edition, and there are versions in 282 languages (as of June 2016). More than 28 million people have contributed to Wikipedia, and more than 116,000 can be considered active users, meaning they have edited content during the last month. It is, therefore, the biggest multilingual collaborative effort ever made (Samoilenko et al., 2016). In the particular case of the Spanish language edition of Wikipedia, it has more than 1.2 million articles and is the $9^{\text {th }}$ largest, with more than 15,000 active users in 2017. According to the Instituto Cervantes, there are almost 468 million people that have Spanish as their mother tongue, thus its importance as a channel for accessing scientific and technological knowledge. Furthermore, most research on Wikipedia focuses exclusively on the English version and studies are needed in other editions (Mesgari et al., 2015). Along this line, Figuerola, Groves, and Quintanilla (2015) have analyzed the Spanish Wikipedia as an educational tool for scientific and technological content, using a completely manual approach in a data dump dated November 2013. Our paper extends the work of Figuerola, Groves, and Quintanilla (2015) by using pre-existing taxonomies for identifying scientific and technological content in a more recent dump dated December 2014.

Wikipedia uses categories to classify articles in a huge pseudo-taxonomy, which has also been collaboratively created (Muchnik et al., 2007; Thornton; McDonald, 2012; Kaptein; Kamps, 2013). Although the quality of content in Wikipedia pages is comparable to other encyclopedias (Lih, 2004), this cannot be said about the taxonomy of categories used to describe its content (Silva et al., 2011; Salah et al., 2012; Hejazy; El-Beltagy, 2013). Several authors have stated problems with the Wikipedia category system. Among them, Capocci, Rao, and Caldarelli (2007) analyzed the structure imposed by categories, showing that it did not reproduce the natural division of articles according to their links. Halavais and Lackaff (2008) showed that Wikipedia coverage depended on its users' interests, as well the category system used to organize them. Holloway, Božicevic, and Börner (2007) described some of the known inconsistencies of categories, such as lack of hierarchical structure or the presence of cycles (Muchnik et al., 2007). As stated in (Suchecki et al., 2012), taxonomies have been more stable in the bottom (that is, the category terms in Wikipedia pages do not change over time) than in the top-level terms, which may be reorganized occasionally. Furthermore, the taxonomy is not consistent across languages, making the creation of multilingual collections for a given concept or domain very difficult. As several authors have pointed out, this is mainly because Wikipedia categories are more a folksonomy rather than a true taxonomy (Jiménez-Pelayo, 2009; Hejazy; El-Beltagy, 2013). Nevertheless, and despite these facts, Wikipedia categories have been extensively used to extract ontological knowledge from Wikipedia (Kaptein; Kamps, 2013; Ryu; Jang; Kim, 2014; Jiang et al., 2015), showing its importance as a huge ground-truth data set for building knowledge-based systems.

However, some precautions should be taken when working with Wikipedia categories. For instance, the Spanish Wikipedia contains a top-level page for categories, including a category for 'Science' (linking to 13 subcategories and 15 pages) and another for 'Technology' (30 subcategories and 187 pages). In the case of 'Science', the 13 subcategories point to 636 subcategories, including non-scientific content (i.e. 'Pseudoscience'). In the case of 'Technology', the 30 subcategories point to 367 subcategories. Furthermore, there are other possible entry points providing access to some of these subcategories, such as 'Academic disciplines', so they cannot be considered to be orthogonal or mutually exclusive. As shown by Suchecki et al. (2012) and Hejazy, and El-Beltagy (2013), some cleansing is needed in order to obtain a valid hierarchized taxonomy.

\section{The Wikipedia categories are more a} folksonomy rather than a true taxonomy

Therefore, we decided not to rely only on categories to analyze the Wikipedia content structure in order to determine scientific and technological content. It would be interesting to identify all the Wikipedia pages related to a given concept, without having to manually review the taxonomy of Wikipedia categories and deciding whether each category and the pages it points to are truly related to that concept or not.

In this paper we describe a semi-automatized method for creating a corpus of Wikipedia articles devoted to scientific and technological content. As we cannot rely on Wikipedia 
taxonomies, we will combine the use of community detection techniques based on random walks, extracting information from the internal structure of Wikipedia links between pages following the approach described by Figuerola, Groves, and Quintanilla (2015), but including the use of existing taxonomies accepted as a de facto standard for describing academic content. The research questions addressed in this paper are:

Q1: Can a significant subset of Wikipedia pages related to scientific and technological content be semi-automatically identified without using the existing category structure?

Q2: Does such subset contain comprehensive coverage of scientific and technological content or is it biased towards some specific fields?

\section{Methodology}

Due to its encyclopedic and hyperlinked nature, most Wikipedia pages link to other pages that can be regarded as being devoted to the same topic, as they explain or introduce related concepts. For instance, 'oxygen' links to 'chemical element', 'atomic number', and 'periodic table', among others. These pages point back to 'oxygen' and other chemical elements as well, creating a dense structure of links between them. Therefore, any random walk starting in 'oxygen' will probably visit several Wikipedia pages about other chemical elements, discovering an underlying community structure (Pons; Latapy, 2005), which can be used to automatically categorize Wikipedia pages. Notice that, throughout this paper, we use "community" as the name for any subset of strongly connected Wikipedia pages that are related to the same topic.

In this paper we describe a semi-automatized method for creating a corpus of Wikipedia articles devoted to scientific and technological content

\subsection{Building the graph}

In our case, we are only interested in detecting the underlying graph structure between pages and links, so we will use only the XML dump file containing Wikipedia pages. We have analyzed a dump corresponding to the Spanish version of Wikipedia, taken on the $17^{\text {th }}$ of December, 2014. The XML file occupies more than nine GB and it contains more than three million pages and 60 million links to other Wikipedia pages.

The first step is to recreate the hyperlinked structure of web pages, which allows Wikipedia users to jump from one page to another. Pages include Wikipedia articles but also categories, lists, user pages, discussion and so on, which cannot be considered real content for the purposes of our analysis. Therefore, if we want to generate a graph containing only pure content, i.e. articles, we need to remove these special pages. On the other hand, we will include annex pages as they are usually lists of Wikipedia pages related to the same concept. This can easily be done as these special pages usually use a particular prefix that identifies them. For instance, categories are Wikipedia pages that always have 'Category:' as a prefix before the category page ('Categoría:' in Spanish). Analogously, we also remove pages containing files, images, templates, and other pages related to Wikipedia (i.e. documentation). Disambiguation pages can also be removed as their names always finish with '_(desambiguación)'. Then we resolve all issues related to redirections, including acronyms, different pages pointing to the same one, spaces, and capital letters, as well as taking care of special characters (like ' $\tilde{\mathrm{N}}$ ' in Spanish) which may have been removed or replaced in order to ensure proper URL functioning. Finally, as two pages can be linked by more than one jump (i.e. the linked page can appear several times in the starting page), we remove all duplicated links but one, maintaining this relationship. We also remove edges linking a page to itself, as they have no meaning for the purposes of our analysis.

\section{Wikipedia can be seen as a scale-free network}

The resulting graph has $1,143,024$ nodes (that is, Wikipedia pages) and 25,469,978 edges (that is, links between nodes). As stated, we did not include 225,380 category pages. Due to its nature, Wikipedia can be seen as a scale-free network (Barabási; Albert, 1999), where the distribution of node out-degrees (i.e. number of links) emerges automatically from a stochastic growth model in which new nodes are added continuously and attach themselves preferentially to existing nodes, with probability proportional to the degree of the target node--so richly connected nodes get richer (Strogatz, 2001), becoming hubs to hundreds or even thousands of pages (like annex pages).

\subsection{Analyzing the graph structure}

Among the several algorithms that can be used to detect communities, we have followed the same approach described in Bohlin et al. (2014), based in the Infomap algorithm (Rosvall; Bergstrom, 2008). Infomap clusters tightly interconnected nodes into modules (two-level clustering) or the optimal number of nested modules (multi-level clustering). It is known to be a fast method that outperforms other algorithms for community detection while providing a high modularity index, which is very suitable for large graphs (Emmons et al., 2016).

For each node in $\mathrm{G}$ (i.e., a page), we obtain a sequence of nested communities and subcommunities that determine the category for that page. The communities form a hierarchical tree, T, with non-overlapping nodes, so each page belongs to a unique community, represented by a leaf on the tree. Obviously, each page also belongs to all other internal nodes that connect the tree root (that contains all communities) with the leaf. Communities are coded $C_{1}: C_{2}: \ldots: C_{L}$ where $L$ is the maximum nesting level, automatically determined by the Infomap algorithm. 
Table 1. 2-digit Unesco taxonomy terms

\begin{tabular}{|c|c|c|c|}
\hline 11 - Logic & 12 - Mathematics & 21 - Astronomy, Astrophysics & 22 - Physics \\
\hline 23 - Chemistry & 24 - Life Sciences & 25 - Earth and Space Science & 31 - Agricultural Sciences \\
\hline 32 - Medical Sciences & 33 - Technological Sciences & 51 - Anthropology & 52 - Demography \\
\hline 52 - Economic Sciences & 54 - Geography & 55 - History & 56 - Juridical Science and Law \\
\hline 57 - Linguistics & 58 - Pedagogy & 59 - Political Science & 61 - Psychology \\
\hline $62-$ Sciences of Arts and Letters & 62 - Sociology & 71 - Ethics & 72 - Philosophy \\
\hline
\end{tabular}

\subsection{Mapping to existing categories}

Once the internal structure of the graph is revealed, it can be mapped to the existing categories used to describe pages in each detected community. Nevertheless, it is not possible to use Wikipedia categories as they are neither mutuaIly exclusive nor collectively exhaustive (Salah et al., 2012). Therefore, we decided to take advantage of a popular taxonomy used to categorize scientific (in a wide sense) content, namely the 'Unesco nomenclature for fields of science and technology' (Unesco, 1988). This taxonomy provides three levels of refinement through a two, four, and six digit-based scheme for coding knowledge domains. There are 24 two-digit taxonomy terms (shown in Table 1), 245 four-digit taxonomy terms, and 2183 six-digit taxonomy terms in the 1988 version. Despite its obsolescence (Unesco abandoned it in 1992), representativeness and usability problems (Martínez-Frías; Hochberg, 2007), it has become a de facto standard for categorizing scientific and technological knowledge, and it constitutes a good starting point (Ruiz-Martínez; Baños-Moreno; Martínez-Béjar, 2014) for many other purposes.

It is not surprising that the Unesco taxonomy also has a page in Wikipedia, although the coverage is different across language versions. In the case of the Spanish Wikipedia, it is possible to retrieve all taxonomy terms from a single entry point, namely the six-digit taxonomy page. For instance, the first term is ' 11 (Logic)', which links to a Wikipedia page containing a link for such page and links to other six four-digit categories, namely '1101 (Application of Logic)', '1102 (Deductive Logic)', '1103 (General Logic)', '1104 (Inductive Logic)', '1105 (Methodology)', and '1199 (Other specialties relating to Logic)'. Four of these four-digit categories also link to Wikipedia pages, although one of them is empty. Within each four-digit term there are zero or more six-digit taxonomy terms, such as '110201 (Analogy)', for instance, which points to a Wikipedia page defining such concept. Unfortunately, this is different in languages other than Spanish, where these pages may not exist. This is a perfect example of the different levels of development that can be found in Wikipedia (Halavais; Lackaff, 2008; Jiménez-Pelayo, 2009; Samoilenko et al., 2016), as content is created according to users' interests, with little coordination.

In the Spanish case, the top-level Unesco taxonomy page points to a total of 1,251 Wikipedia pages, although unfortunately some of them are empty web pages, point to wrong pages, or to repeated ones. Once cleansed, the Wikipedia Unesco taxonomy points to 974 valid Wikipedia pages, that is, $44.6 \%$ of the total six-digit Unesco categories.

\section{Results}

We have used the available Infomap implementation as described in Bohlin et al. (2014), using default values for the input parameters. Infomap is known to be one of the best algorithms for this purpose (Lancichinetti; Fortunato, 2009). Nevertheless, as stated in Emmons et al. (2016), Infomap can fail to identify large communities in very large networks, as in our case. On the other hand, Infomap may also fail to generate accurate communities if there are too many small ones, as also occurs in our case. These two issues mean that:

a) several pages from the same topic might fall in different communities; and

b) small communities might not be detected at all.

\subsection{Communities detected}

We obtained 35,296 communities and subcommunities (up to five nesting levels), containing between 1 and 17,588 pages, following a long-tail distribution, as only 1,442 communities included 100 or more Wikipedia pages, containing $79.1 \%$ of all the Wikipedia pages used to create the graph. If we want to include at least $90 \%$ of all the Wikipedia pages in the analysis, we would need to analyze 3,662 communities containing 32 or more pages. In theory, each one of these 3,662 communities should be manually inspected, in order to determine the main topic. For instance, the largest community (coded by the Infomap algorithm as ' $1: 1$ ') contains 17,588 pages about movies, actors and actresses, directors, and cinema in general. The second one (coded as ' $1: 15^{\prime}$ ') contains 13,633 pages about Chile, the country. On the other hand, the third largest community (coded as '2:2:6') contains 13,420 pages about the order 'Coleoptera', which can be considered scientific content. Obviously, manually examining 3,662 communities is not reasonable, so we need to use another approach that reduces the number of communities to inspect to a reasonable amount. Figuerola, Groves and Quintanilla (2015) describe a similar approach, but they only analyze the 255 largest top-level communities detected by Infomap in an older dump (November 2013), without describing other detaiIs of the manual review process. Furthermore, they use the Wikipedia dump without removing special pages such as categories, which were discarded in our analysis because of the aforementioned inconsistencies. Table 2 summarizes the structure of the most important communities and subcommunities for the top levels. The distribution of communities found by Figuerola, Groves, and Quintanilla (2015) was completely different, although the authors also identified long tails with very small communities. 
Instead of analyzing each community and trying to determine whether it can be considered part of the science and technology corpus or not, following a topdown approach, we will use a bottom-up approach, starting from the Unesco six-digit taxonomy pages. The other pages in the same community will then also be assumed to belong to this category, bounding the probability of misclassification by means of sampling analysis.

\subsection{Mapping to the Unesco taxonomy}

The Unesco nomenclature is present in Wikipedia as a three-level hierarchical taxonomy, as described in Ruiz-Martínez, Baños-Moreno and Martínez-Béjar (2014). There is a Wikipedia page for two-digit Unesco terms pointing to other pages containing four-digit terms, which in turn point to pages containing six-digit terms. These Wikipedia pages also include links to other Wikipedia pages with content describing the concepts used in the Unesco nomenclature. Therefore, starting from the Wikipedia page describing the two-digit Unesco nomenclature, 974 different Wikipedia pages can be reached.

For each one of these 974 Wikipedia pages, we have identified the community that the page belongs to, using the results provided by the Infomap algorithm, which assigns a community for each page. These 974 pages can be mapped to 458 different communities, containing a total of 205,907 Wikipedia pages, that is, $18.0 \%$ of the total number of Wikipedia pages considered to be content articles. Nevertheless, we must ensure that all pages reachable from the Unesco six-digit taxonomy page are truly scientific and technological content. For instance, all communities containing pages related to artistic works (music, paintings, etc.) or geographical places were discarded.

\section{The Unesco nomenclature is present in Wikipedia as a three-level hierarchical taxonomy}

For this purpose, the 458 identified communities were then manually inspected in order to determine whether they could really be considered scientific and technological content or not. We followed a procedure derived from a single-sampling acceptance plan (Ruggeri; Kenett; Faltin, 2007), treating communities as 'lots', but taking into account the large variability of community size, thus establishing criteria for small and large communities. In order to do so, each community was inspected by two researchers (out of five), according to the following criteria:

- If the community was small (i.e. less than or equal to 125 articles, a total of 302 communities out of 458 ), it was completely screened by searching for content not considered to be scientific or technological content, measuring the percentage of false positives. Communities with more than $15 \%$ of false positives were rejected.

- If the community was too large for complete screening, a tag cloud containing all the words in the pages' titles was built for preliminary analysis purposes, detecting communities with unexpected terms that could be rapidly discarded. For instance, communities containing pages about cities or geographical regions fell into this category. Additionally, a subset of 125 articles was further inspected, discarding the community if more than $20 \%$ of false positives was found. This is equivalent to an inspection level II single sampling acceptance plan.

- If the two researchers did not agree on whether a community should belong to the scientific and technological corpus or not, it was then inspected once again by all the researchers, reaching a consensus decision.

- Decisions were made at the community level, so no individual pages were discarded or included in the corpus.

This process generated a final corpus of 340 communities containing a total of 60,108 Wikipedia pages. This is only $5.3 \%$ of the total number of Wikipedia pages considered, but all of them can be considered to be content related to science and technology, with almost no false positives. After cleansing, Figuerola, Groves and Quintanilla (2015) found 119,797 Wikipedia pages that were considered to contain scientific and technological content (11.7\%), although the 
Table 3. Number of pages belonging to each Unesco two-digit taxonomy term

\begin{tabular}{|c|c|c|c|c|c|c|c|c|}
\hline $\begin{array}{l}\text { Unesco } \\
\text { 2-digit term }\end{array}$ & 33 & 24 & 21 & 25 & 22 & 12 & 23 & 63 \\
\hline Number of pages & 13,986 & 8,598 & 8,253 & 5,386 & 4,329 & 3,829 & 2,425 & 2,260 \\
\hline$\%$ & 23.3 & 14.3 & 13.7 & 9.0 & 7.2 & 6.4 & 4.0 & 3.8 \\
\hline $\begin{array}{l}\text { Unesco } \\
\text { 2-digit term }\end{array}$ & 72 & 32 & 53 & 61 & 31 & 57 & 51 & 59 \\
\hline Number of pages & 1,936 & 1,701 & 1,604 & 985 & 985 & 928 & 900 & 744 \\
\hline$\%$ & 3.2 & 2.8 & 2.7 & 1.6 & 1.6 & 1.5 & 1.5 & 1.2 \\
\hline $\begin{array}{l}\text { Unesco } \\
\text { 2-digit term }\end{array}$ & 55 & 11 & 52 & 56 & 54 & 71 & 58 & 62 \\
\hline Number of pages & 344 & 336 & 297 & 144 & 77 & 61 & 0 & 0 \\
\hline$\%$ & 0.6 & 0.6 & 0.5 & 0.2 & 0.1 & 0.1 & 0.0 & 0.0 \\
\hline
\end{tabular}

authors did not bound the possible misclassification error when considering each community as a whole.

In light of these results and answering question Q1, we can say that it is possible to extract scientific and technological content without using the existing category structure, and we provide a method for this task.

It is remarkable that from the 24 Unesco two-digit taxonomy terms (shown in Table 1), 22 appear in the corpus. The two terms that do not appear in the corpus are '58 (Pedagogy)' and ' 62 (Sciences of Arts and Letters)'. The latter was not considered to be part of the corpus, as previously mentioned. On the other hand, the former is a Wikipedia page with no links to other pages, thus becoming an isolated page not traversed by the Infomap algorithm and, therefore, not belonging to any relevant community. On the other hand, as shown in Table 3, the six most represented Unesco two-digit taxonomy terms are 'Technological Sciences' (33), 'Life Sciences' (24)', 'Astronomy, Astrophysics' (21), 'Earth and
Space Sciences' (25), 'Physics' (22) and 'Mathematics' (12), accounting for more than $73.9 \%$ of the total pages considered scientific and technological content.

Figure 1 shows a graph describing how Unesco communities are related to each other according to the number of links they share, that is, their similarity in a distance sense. This graph was created with VOS (Van-Eck; Waltman, 2007). Nodes represent Unesco categories and edges represent links between pages belonging to two categories. Node size is proportional to the number of pages in that category, while edge width is proportional to the number of links shared between those communities. VOS identifies three large clusters of communities, each one of them coded in a different color.

Notice that there is a clear bias towards technological content (the largest nodes), while social sciences or medicine are clearly underrepresented, as stated by Halavais and Lackaff (2008) and more recently in Mesgari et al. (2015). Ne-

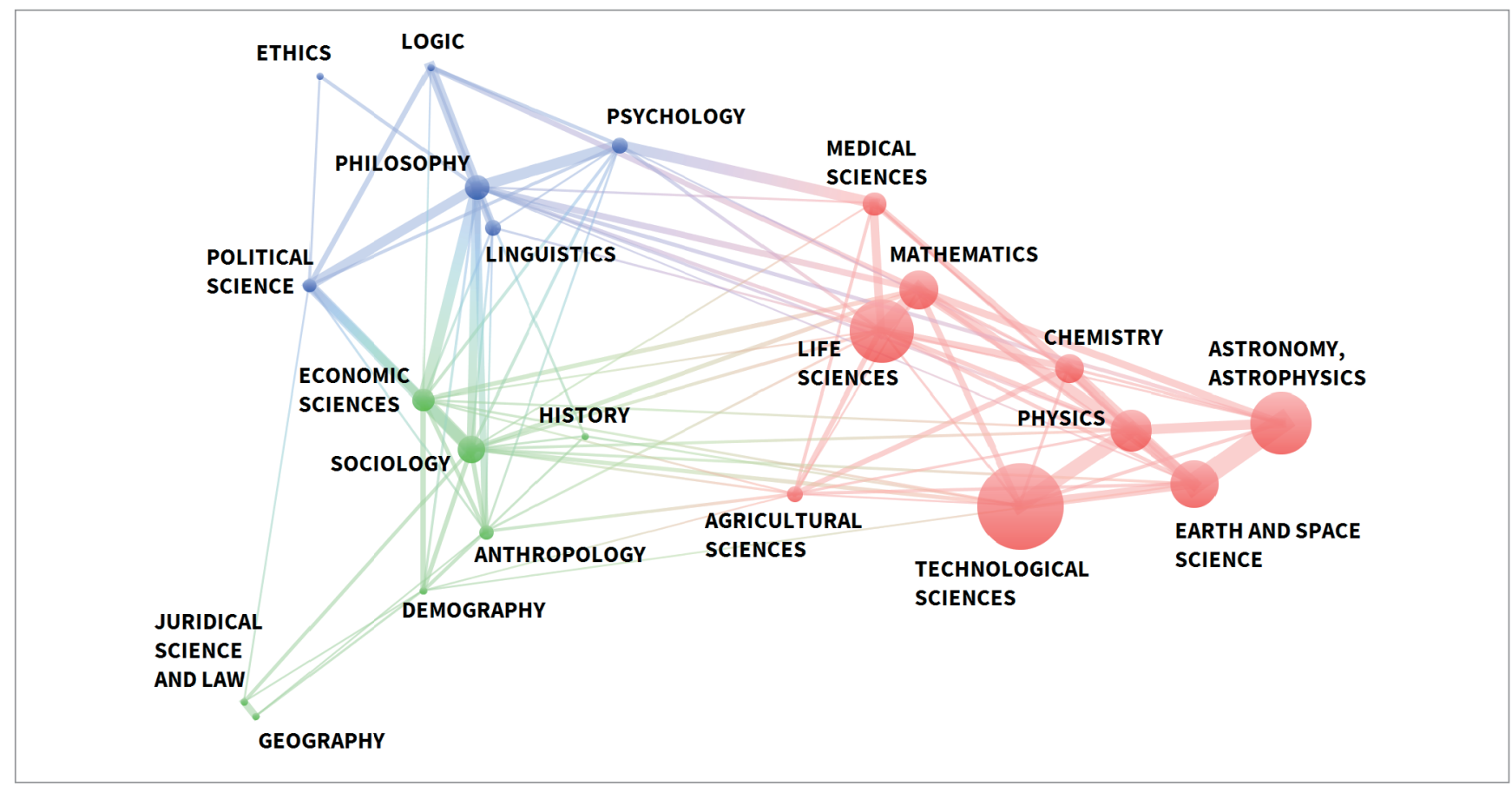

Figure 1. VOS map of Unesco communities according to the number of links they share within the scientific and technological corpus. 
vertheless, there are some similarities with standard cognitive maps of science, as the one proposed by Rafols, Porter and Leydesdorff (2010): a quasi-modular and fragmented structure with some noticeable clusters and without a clear center. The clusters identified by VOS are also comparable to those described in Rafols, Porter and Leydesdorff (2010), as well as the main edges between the most important nodes within and between clusters. For instance, there is a strong edge connecting "Astronomy, Astrophysics" with "Earth and Space Science" and another one connecting "Psychology" with "Medical Sciences".

Therefore, answering question Q2, our method produces a comprehensive corpus across disciplines and quite consistent with contemporary global images of science, although it also reflects the known bias of Wikipedia towards technological content.

\section{Wikipedia has a clear bias towards te- chnological content (the largest nodes), while social sciences or medicine are clearly underrepresented}

\section{Conclusions}

Wikipedia has recently become -among many other thingsthe main source of scientific information for the general public and it is increasingly used in academia, by students and faculty (Aibar et al., 2015). Recent studies on communication and public perception of science in several countries agree that the Internet has turned out to be, for most people, the main source of scientific information and that within the Internet, Wikipedia is the single most browsed site. People use it not only to satisfy their curiosity on the Higgs boson or on special relativity, but also to learn about more 'sensitive' issues of health and medical conditions that might be used to make practical decisions. Nonetheless, scientists and scientific institutions do not seem to be aware of this situation. Therefore, it is important to analyze the nature of scientific and technological content in Wikipedia, which needs to be identified by other means than the current categories, which have not been systematically created.

Taking advantage of the highly interlinked structure of Wikipedia, we used a graph random walk algorithm for detecting communities of pages strongly linked to each other. The original Wikipedia dump was pre-processed to generate a graph, removing duplicates, special pages, and resolving language related issues. A total of 60,108 Wikipedia pages distributed in 340 communities were identified as scientific and technological content. These pages belong to those communities that are reachable from the 974 Unesco six-digit taxonomy terms, removing those communities that cannot be considered scientific or technological. From the original 24 categories in the Unesco two-digit taxonomy, 22 are represented in the corpus, with a clear bias towards technology and natural sciences. The resulting graphical layout of the relationships between the different fields -as a function of the intern links between the articles within them- shows a coherent picture when compared to standard cognitive maps of science and a high correlation with other databases such as Scopus and Web of Science.

This small subset (5.3\% of the total Spanish Wikipedia pages) is, nevertheless, a valuable resource for analyzing differences in Wikipedia as a mechanism for transmitting scientific and technological content compared to common knowledge. It also shows the large amount of scientific and technological content in Wikipedia that can be semi-automatically discovered, which is probably larger and could be extended by other means. Wikipedia is, therefore, a great portal for introducing people to scientific and technological content, establishing a bridge between society and academia.

Besides obtaining the scientific and technological corpus, the results of this analysis could be directly applied to Wikipedia, as up to three new categories could be added to all the pages considered to be part of it, describing the Unesco two-digit, four-digit, and six-digit taxonomies that the page belongs to. On the other hand, some of the detected communities have no link to the Unesco taxonomy pages, so they could also be improved by adding these new terms. Finally, the Unesco taxonomy could be recreated as a Wikipedia category structure and linked to the general "Science" category, helping Wikipedia users to find scientific and technological content. Providing a better support for categories would encourage increased participation in the expansion and refinement of the category system, especially among novice editors, as stated in Thornton \& McDonald (2012).

\section{The Unesco taxonomy could be recrea- ted as a Wikipedia category structure and linked to the general "Science" ca- tegory, helping Wikipedia users to find scientific and technological content}

Current and future research on this topic should take into account two important dimensions in Wikipedia: temporal, as it is an evolving environment, thus promoting longitudinal studies; and idiomatic, as different languages may show distinct cultural approaches towards scientific and educational content. Once a scientific and technological corpus has been established, it is possible to analyze, among other things, whether Wikipedia shows an accurate representation of present scientific knowledge -comparing the distribution of articles with standard repositories of scientific output- or not, as well as whether mainstream positions tend to be favored in dealing with controversial issues, and the kind of sources that are used by editors.

\section{Acknowledgements}

This research is part of the project "Análisis del contenido científico de la Wikipedia española", funded by Fecyt, ref. FCT-14-8269.

\section{References}

Aibar, Eduard; Lladós-Masllorens, Josep; Meseguer-Artola, Antoni; Minguillón, Julià; Lerga, Maura (2015). “Wikipedia 
at university: what faculty think and do about it". The electronic library, v. 33, n. 4, pp. 668-683.

http://openaccess.uoc.edu/webapps/o2/handle/10609/39442 https://doi.org/10.1108/EL-12-2013-0217

Barabási, Albert-László; Albert, Réka (1999). "Emergence of scaling in random networks". Science, v. 286, pp. 509-512. http://barabasi.com/f/67.pdf

https://doi.org/10.1126/science.286.5439.509

Bohlin, Ludvig; Edler, Daniel; Lancichinetti, Andrea; RosvaII, Martin (2014). "Community detection and visualization of networks with the map equation framework". In: Ding, Ying; Rousseau, Ronald; Wolfram, Dietmar (eds.). Measuring scholarly impact. Springer International Publishing, pp. 3-34. https://doi.org/10.1007/978-3-319-10377-8_1

Brossard, Dominique; Scheufele, Dietram (2013). "Science, new media, and the public". Science, v. 339, n. 6115, pp. 40-41. https://goo.gl/9W5zZR

https://doi.org/10.1126/science.1232329

Capocci, Andrea; Rao, Francesco; Caldarelli, Guido (2007). "Taxonomy and clustering in collaborative systems: The case of the on-line encyclopedia Wikipedia". Europhysics letters, v. 81, n. 2.

https://arxiv.org/abs/0710.3058

https://doi.org/10.1209/0295-5075/81/28006

Emmons, Scott; Kobourov, Stephen; Gallant, Mike; Börner, Katy (2016). "Analysis of network clustering algorithms and cluster quality metrics at scale". PLoS one, v. 11, n. 7, art. no. e0159161.

https://doi.org/10.1371/journal.pone.0159161

Ermann, Leonardo; Frahm, Klaus M.; Shepelyansky, Dima L. (2015). "Google matrix analysis of directed networks". Reviews of modern physics, v. 87, n. 4.

https://arxiv.org/abs/1409.0428

https://doi.org/10.1103/RevModPhys.87.1261

Figuerola, Carlos G.; Groves, Tamar; Quintanilla, Miguel-Ángel (2015). "The implications of Wikipedia for contemporary science education: Using social network analysis techniques for automatic organisation of knowledge". In: Proceedings of the $3^{\text {rd }}$ Int l conf on technological ecosystems for enhancing multiculturality, TEEM'15, Porto, October 7-9, pp. 403-410.

http://eprints.rclis.org/29277/1/20156 figue.pdf

https://doi.org/10.1145/2808580.2808641

Halavais, Alexander; Lackaff, Derek (2008). "An analysis of topical coverage of Wikipedia". Journal of computer-mediated communication, v. 13, n. 2, pp. 429-440.

https://doi.org/10.1111/j.1083-6101.2008.00403.x

Hejazy, Khaled A.; El-Beltagy, Samhaa R. (2013). "An approach for deriving semantically related category hierarchies from Wikipedia category graphs". In: Rocha, Álvaro; Correia, Ana-María; Wilson, Tom; Stroetmann, Karl A. (eds.). Advances in information systems and technologies. Berlin Heidelberg: Springer, pp. 77-86.

Holloway, Tod; Božicevic, Miran; Börner, Katy (2007). "Analyzing and visualizing the semantic coverage of Wikipedia and its authors". Complexity, v. 12, n. 3, pp. 30-40. http://nwb.cns.iu.edu/papers/holloway-0000-analvizwikipedia. $p d f$

https://doi.org/10.1002/cp/x.20164

Jiang, Yuncheng; Zhang, Xiaopei; Tang, Yong; Nie, Ruihua (2015). "Feature-based approaches to semantic similarity assessment of concepts using Wikipedia". Information processing \& management, v. 51, n. 3, pp. 215-234.

https://doi.org/10.1016/j.ipm.2015.01.001

Jiménez-Pelayo, Jesús (2009). “Wikipedia como vocabulario controlado: ¿está superado el control de autoridades tradicional". El profesional de la información, v. 18, n. 2, pp. 188-201. https://doi.org/10.3145/epi.2009.mar.09

Kaptein, Rianne; Kamps, Jaap (2013). "Exploiting the category structure of Wikipedia for entity ranking". Artificial intelligence, v. 194, pp. 111-129.

https://doi.org/10.1016/j.artint.2012.06.003

Kim, Kyung-Sun; Sin, Sei-Ching-Joanna; Yoo-Lee, Eun-Young (2014). "Undergraduates' use of social media as information sources". College \& research libraries, v. 75, n. 4, pp. 442-457. https://doi.org/10.5860/crl.75.4.442

Lancichinetti, Andrea; Fortunato, Santo (2009). "Community detection algorithms: A comparative analysis". Physical review $E$ - statistical, nonlinear, and soft matter physics, v. 80 , n. 5, art. no. 056117.

https://arxiv.org/abs/0908.1062

https://doi.org/10.1103/PhysRevE.80.056117

Lih, Andrew (2004). "Wikipedia as participatory journalism: Reliable sources? Metrics for evaluating collaborative media as a news resource". In: Procs of the $5^{\text {th }}$ Int/ symposium on online journalism, pp. 16-17.

https://goo.gl/6XpLn9

Martínez-Frías, Jesús; Hochberg, David (2007). “Classifying science and technology: two problems with the Unesco system". Interdisciplinary science reviews, v. 32, n. 4, pp. 315-319. http://digital.csic.es/handle/10261/13013

Medelyan, Olena; Milne, David; Legg, Catherine; Witten, Ian (2009). "Mining meaning from Wikipedia". International journal of human-computer studies, v. 67, n. 9, pp. 716-754. https://arxiv.org/abs/0809.4530

https://doi.org/10.1016/j.ijhcs.2009.05.004

Mesgari, Mostafa; Okoli, Chitu; Mehdi, Mohamad; Nielsen, Finn-Årup; Lanamäki, Arto (2015). "'The sum of all human knowledge': A systematic review of scholarly research on the content of Wikipedia". Journal of the Association for Information Science and Technology, v. 66, n. 2, pp. 219-245. http://spectrum.library.concordia.ca/978618/ https://doi.org/10.1002/asi.23172

Muchnik, Lev; Itzhack, Royi; Solomon, Sorin; Louzoun, Yoram (2007). "Self-emergence of knowledge trees: Extraction of the Wikipedia hierarchies". Physical review E, v. 76, n. 1. https://doi.org/10.1103/PhysRevE.76.016106

Pons, Pascal; Latapy, Matthieu (2005). "Computing communities in large networks using random walks". In: Yolum, Pinar; Güngör, Tunga; Gürgen, Fikret; Özturan, Can (eds.). Procs of the $20^{\text {th }}$ Intl symposium on computer and information sciences (ISCIS 2015). Berlin, Heidelberg: Springer, pp. 284-293. 
https://arxiv.org/abs/physics/0512106

Ponzetto, Simone-Paolo; Strube, Michael (2007). "Knowledge derived from Wikipedia for computing semantic relatedness". Journal of artificial intelligence research, v. 30, pp. 181-212.

https://doi.org/10.1613/jair.2308

Rafols, Ismael; Porter, Alan L.; Leydesdorff, Loet (2010). "Science overlay maps: A new tool for research policy and library management". Journal of the American Society for information Science and Technology, v. 61, pp. 1871-1887. http://www.leydesdorff.net/overlaytoolkit/overlaytoolkit.pdf https://doi.org/10.1002/asi.21368

Rosvall, Martin; Bergstrom, Carl T. (2008). "Maps of random walks on complex networks reveal community structure". In: Procs of the National Academy of Sciences, v. 105, n. 4, pp. 1118-1123.

https://doi.org/10.1073/pnas.0706851105

Ruggeri, Fabrizio; Kenett, Ron S.; Faltin, Frederick (eds.) (2007). Encyclopedia of statistics in quality and reliability. Wiley. ISBN: 9780470061572

https://doi.org/10.1002/9780470061572

Ruiz-Martínez, Juana-María; Baños-Moreno, María-José; Martínez-Béjar, Rodrigo (2014). "Nomenclatura Unesco: evolución, alcance y reutilización en clave ontológica para la descripción de perfiles científicos". El profesional de la información, v. 23, n. 4, pp. 383-392.

https://doi.org/10.3145/epi.2014.jul.06

Ryu, Pum-Mo; Jang, Myung-Gil; Kim, Hyun-Ki (2014). "Open domain question answering using Wikipedia-based knowledge model". Information processing \& management, v. 50 , n. 5 , pp. 683-692.

https://doi.org/10.1016/j.ipm.2014.04.007

Salah, Almila-Akdag; Gao, Cheng; Suchecki, Krzysztof; Scharnhorst, Andrea (2012). "Need to categorize: A comparative look at the categories of universal decimal classification system and Wikipedia". Leonardo, v. 45, n. 1, pp. 84-85. https://arxiv.org/abs/1105.5912

https://doi.org/10.1162/LEON_a_00344

Samoilenko, Anna; Karimi, Fariba; Edler, Daniel; Kunegis, Jérôme; Strohmaier, Markus (2016). "Linguistic neighbour- hoods: Explaining cultural borders on Wikipedia through multilingual co-editing activity". EPJ data science, v. 5, n. 9. https://doi.org/10.1140/epjds/s13688-016-0070-8

Silva, Filipi-Nascimento; Viana, Matheus-Palhares; Travençolo, Bruno A. N.; Costa, Luciano da F. (2011). "Investigating relationships within and between category networks in Wikipedia". Journal of informetrics, v. 5, n. 3, pp. 431-438.

https://goo.gl/6wF8cR

https://doi.org/10.1016/j.joi.2011.03.003

Snyder, Johnny (2013). “Wikipedia: Librarians' perspectives on its use as a reference source". Reference \& user services quarterly, v. 53, n. 2, pp. 155-163.

https://doi.org/10.5860/rusq.53n2.155

Strogatz, Steven H. (2001). "Exploring complex networks". Nature, n. 410, pp. 268-276.

https://doi.org/10.1038/35065725

Suchecki, Krzysztof; Salah, Alkim-Almila-Akdag; Gao, Cheng; Scharnhorst, Andrea (2012). "Evolution of Wikipedia's category structure". Advances in complex systems, v. 15 (supp01), 1250068.

https://arxiv.org/abs/1203.0788

https://doi.org/10.1142/S0219525912500683

Thornton, Katherine; McDonald, David W. (2012). "Tagging Wikipedia: collaboratively creating a category system". In: Procs of the $17^{\text {th }}$ ACM Intl conf on supporting group work, pp. 219-228.

http://www.pensivepuffin.com/dwmcphd/papers/Thornton_ McDonald-TaggingWikipedia-GROUP12.pdf https://doi.org/10.1145/2389176.2389210

Unesco (1988). Proposed international standard nomenclature for fields of science and technology. NS/ROU/257 REV.1; SC.88/WS/80.

http://unesdoc.unesco.org/images/0008/000829/082946eb. $p d f$

Van-Eck, Nees-Jan; Waltman, Ludo (2007). "VOS: A new method for visualizing similarities between objects". In: Decker, Reinhold; Lenz, Hans J. (eds.). Advances in data analysis: Procs of the $30^{\text {th }}$ Annual conf of the German Classification Society, Berlin, March 8-10. Springer, pp. 299-306. https://doi.org/10.1007/978-3-540-70981-7_34

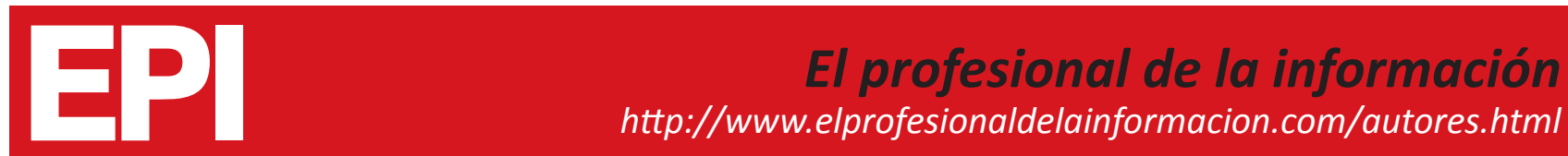

PRÓXIMOS TEMAS

\begin{tabular}{|c|c|l|c|}
\hline Número & Mes año & \multicolumn{1}{|c|}{ Tema } & Envío textos \\
\hline 26,6 & Nov 2017 & Diseño de la información & \\
\hline 27,1 & Ene 2018 & Información personal y datos masivos & 10 nov 2017 \\
\hline 27,3 & Mar 2018 & Indicadores & 10 ene 2018 \\
\hline 27,4 & May 2018 & Información política y redes sociales & 10 mar 2018 \\
\hline 27,5 & Jul 2018 & Posverdad y credibilidad de la información & 10 may 2018 \\
\hline
\end{tabular}


